\title{
Detection of Looping During Colonoscopy Using Bending Sensors
}

\author{
JungHun Choi ${ }^{1, *}$ and David Drozek ${ }^{2}$ \\ ${ }^{I}$ Department of Mechanical Engineering and Biomedical Engineering Program, Ohio University, Athens, Ohio, U.S.A \\ ${ }^{2}$ Department of Specialty Medicine, Heritage College of Osteopathic Medicine, Ohio University, Athens, Ohio, U.S.A
}

\begin{abstract}
During colonoscopy, looping of the colonoscope shaft is considered one of the biggest challenges of the procedure. It hinders the advancement of the distal tip of the colonoscope requiring time to retract and straighten the shaft. Consequently, anesthesia exposure and operative time, and associated risk and cost are all increased. Many active and passive auxiliary devices have been introduced to overcome looping problems but only select devices were utilized due to safety, complexity, or cost issues. In this study, a low cost looping detection system embedded in the shaft of the colonoscope and the corresponding software algorithm have been evaluated. Thirty bending sensors were inserted inside the shaft of the colonoscope, which sent voltage signals to the analog-digital converter. Digital signals were transmitted to the computer for software analysis of the looping status of the colonoscope shaft. A colonoscopist can often detect the beginning of the looping process and can initiate maneuvers to correct and avoid the looping, which frequently are successful. A standard colonoscopy training model was utilized to test the looping detection system, which effectively demonstrated loop formation, providing data to the endoscopist that is helpful for initiation of appropriate loop avoidance techniques. Maintenance of the bending sensors and a learning curve of the system can be potential limitations.
\end{abstract}

Keywords: Colonoscopy, sensor, magnetic endoscope imaging, Looping.

\section{INTRODUCTION}

Colonoscopy is the most common and effective diagnostic procedure to evaluate colorectal diseases in the lower GI (GastroIntestinal) tract. During colonoscopy, looping of the colonoscope shaft is considered one of the biggest challenges of the procedure, at times hindering visualization of the entire colon [1, 2]. In one study, looping occurred in 91 of 100 patients; the $\mathrm{N}$ type loop configuration was most commonly encountered, present in 79\% [3]. Looping increases the discomfort of the procedure for the patient, requiring higher levels of anesthesia, and prolongs the duration of the procedure, increasing the exposure time to anesthesia and its associated risks. Looping causes pain by stretching the mesentery, the tethering structure of the colon $[1,4]$. To escape the loop, the colonoscopist exerts force by pushing, pulling, and twisting the shaft of the colonoscope, risking damage to the mucosa, or inner lining, of the colon [5-7]. Splenic injury is rare, but has been reported with colonoscopy $[8,9]$, associated with blunt and mucosal trauma to the colon [10]. Serosal, or outer lining of the colon, tears and mechanical trauma were also reported during colonoscopy secondary to excessive force exerted by the distal tip of the colonoscope, and corresponding stretch of the colon wall $[11,12]$. Looping can be minimized by introducing some form of external equipment and an active or passive looping prevention system. Looping prevention implies that the colonoscopist can avoid or escape looping using a device which can be at

*Address correspondence to this author at the Department of Mechanical Engineering and Biomedical Engineering Program, Ohio University, Athens, Ohio, U.S.A; Tel: 740-593-2739; Fax: 740-593-0476;

E-mail: choij1@ohio.edu tached to or installed within the colonoscope. Looping prevention devices already exist, such as a) a double balloon sheath near the distal tip, b) a variable stiffness tube, c) ShapeLock Technology, and d) a general overtube for the guidance of the shaft of the colonoscope. Visualization devices such as CT (Computerized Tomographic) colonography, fluoroscopy, and magnetic endoscope imaging can also be utilized as an active looping prevention system. Passive looping prevention methods can be employed to escape or minimize looping when encountered during the intubation. Pre-education for understanding the colonic anatomy and mechanism of looping via colonoscopy simulators, can be helpful in teaching passive loop escape techniques, such as water injection, external abdomen pressure, and patient positioning.

Simple devices can be directly installed to the conventional colonoscope or used as an auxiliary device to overcome the looping. A three component sliding tube, double balloon methods, and sigmoid stiffener are the example of these devices [13-16]. ShapeLock technology was developed for the rapid reinsertion of the colonoscope after full withdrawal [1720]. External devices aid visualization of the configuration of the inserted colonoscope. CT colonography can reveal important anatomic factors that contribute to looping $[4,21]$. Fluoroscopy is also a useful option to visualize colonoscope configuration during difficult procedures [4, 22, 23]. Major pain episodes during colonoscopy were studied using real-time magnetic endoscope imaging (MEI). A magnetic imaging probe was inserted through the biopsy channel. Colonoscope position, configuration and their relationship to pain were evaluated. [24, 25]. MEI was especially helpful during initial training of the colonoscopist, as well as during the process of learning advanced colonoscopic techniques. [26]. 


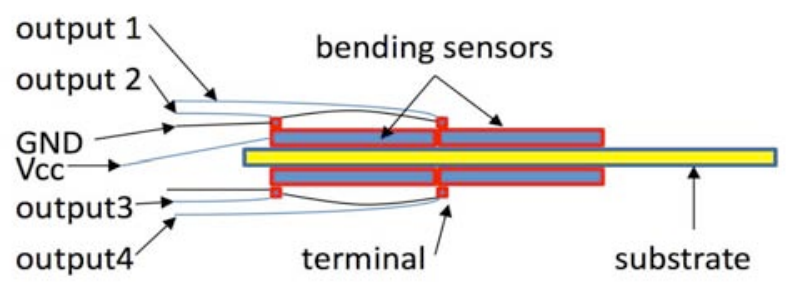

Fig. (1). Example of four bending sensors attached to both sides of the substrate. The excitation voltage is supplied to the bending sensors, and each output signal is connected to the buffer circuit.

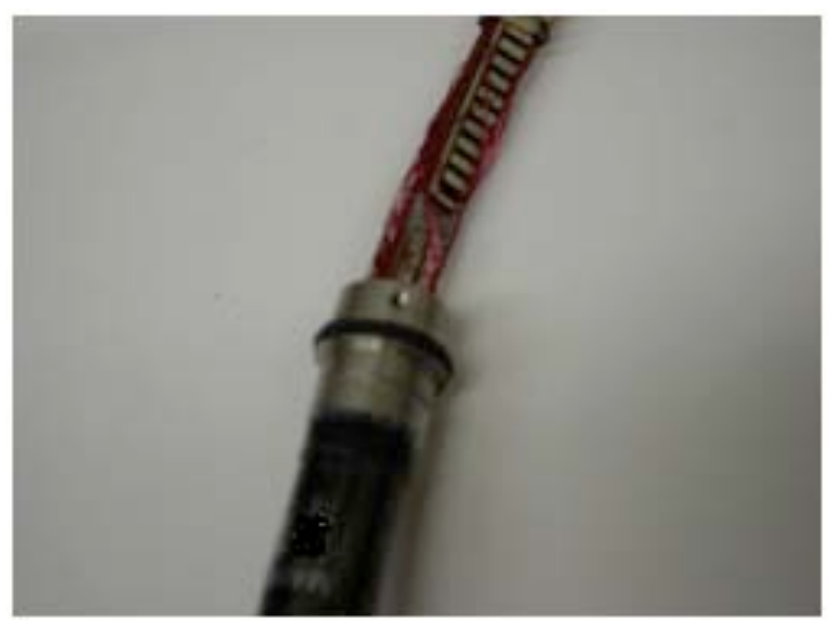

Fig. (2). Serial connection of 15 bending sensors on both sides of a substrate in the shaft of the colonoscope.

A water intubation technique, which instilled water in the first curve of the sigmoid colon during difficult left-side colonoscopies, proved to be safe, decreasing intubation time, regardless of the loop shape (alpha or N) [27]. Patient selfadministered external abdominal pressure was compared with assistant-administered external abdominal pressure to increase the cecal intubation and minimize the loop formation. There was no difference between the two methods. The most commonly helpful location of pressure application was the left lower quadrant of the abdomen [28]. Some intubation techniques were developed to reduce looping occurring during sedation for colonoscopy. Factors increasing the technical difficulty of colonoscopy included: low body mass index, poor bowel preparation, prior abdominal surgical, patient pain, and the diagnosis of irritable bowel syndrome. The prone 12 o'clock patient position and the conventional 6 o'clock left lateral position were compared to determine which was most advantageous. The prone position showed superior results for ileal intubation. [29-31]. To improve the performance in an endoscopy unit, the quality of colonoscopy was assessed. In five hundred procedures cecal intubation rate, bowel preparation, colonoscopy procedures, neoplasia presence, morbidity, and patient satisfaction were analyzed. Deficiencies were identified, leading to quality improvement in the unit. [32]. The colonoscopy learning curve and factors associated with development of competency and proficiency were evaluated. Level of experience, rate of successful completion of the procedure, and cecal intubation time were recorded. The findings of the study implied that two hundred procedures are required to reach a stable point on the learning curve [33].
Various colonoscopy simulators have been utilized to achieve competency and proficiency, decreasing the learning curve more safely and rapidly. From a simple flat-board model, a computer-based force feedback model has been developed. A variety of scenarios and variations of colonic anatomy were provided for the novice colonoscopist [34]. A good colonoscopy simulator should be able to discriminate between the novice, intermediate, and expert colonoscopists. An HT Immersion Medical Colonoscopy Simulator provided different levels of complexity for the different levels of the users [35]. NeoGuide Endoscopy System is a computercontrolled simulator, designed to minimize looping and the need of sedation [36]. Realistic GI endoscopy training methodologies increased the efficiency of the endoscopist to perform therapeutic procedures, such as control of nonvariceal upper GI bleeding, polypectomy, stricture dilation, and percutaneous endoscopic gastrostomy tube insertion [37]. The Olympus colonoscopy simulator provides a high-fidelity training platform compared to the standard patient-based colonoscopy training for the novice colonoscopists. Post training assessment with feedback demonstrated improvement in the general skill level of conventional colonoscopy [38].

Literature reviews clearly demonstrate that looping is a major contributor to prolonged colonoscopy procedure time and decrease success rate. Techniques and devices that avoid or limit looping improve time and success of colonoscopy. In this study, a training model for colonoscopy and a looping detection system consisting of a series of bend sensors inside the shaft of the colonoscope, was implemented to study the intubation time of colonoscopy with the device compared to that without the device.

\section{METHOD}

Uni-directional bend sensors are used to detect the amount of bending. Based on the degree of bending, the bending sensor changes the resistance values which can be converted to voltage. The uni-directional bend sensors can predict the radius of curvature of the shaft in one axis. A total of 30 bend sensors were used to detect the loop formation of the colonoscope. Two sets of fifteen bend sensors were placed between the continuous substrate to identify both sides of bending. Fig. (1) shows parts of bending sensor sets with connections. Fig. (2) shows the end of the shaft and inserted series of bend sensors. In order to install the bend sensor sets inside the shaft of the colonoscope, all the internal components of the shaft of the colonoscope were detached from the base of the colonoscope. After the bending sensor sets were inserted, all the components such as biopsy channel, four tendons to control the distal tip of the colonoscope, image sensor lines, and fiber optic lines were reassembled.

Each bend sensor has two terminals: one connected to the excitation voltage and another to the bend sensor output. A simple buffer circuit using an operational amplifier is used per channel to match the impedance with the data acquisition system and increase stability of the output signal. Each sensor output was adjusted and fine tuned by the individual potentiometer at the buffer circuits to generate the equal outputs. A thirty channel analog-to-digital converter was used to connect all the outputs from the thirty bend sensors. 


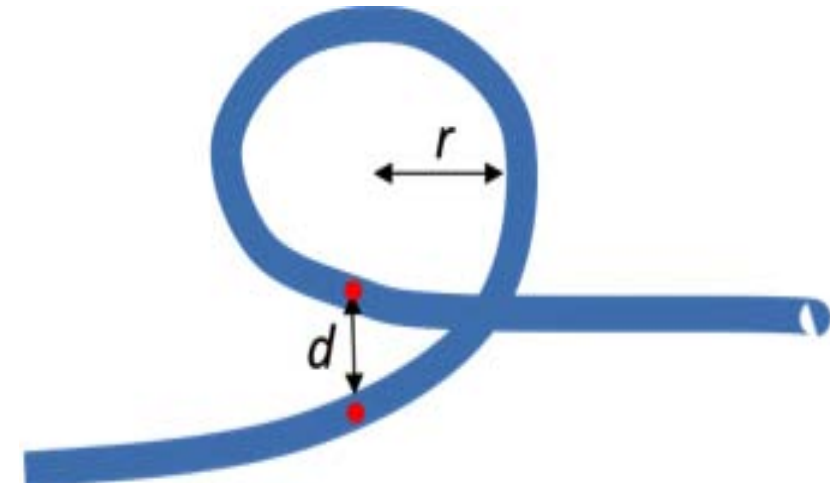

Fig. (3). Typical alpha (or reversed alpha) shape loop with parameters. $r$ is radius of curvature of the shaft and $d$ is three dimensional loop parameter.

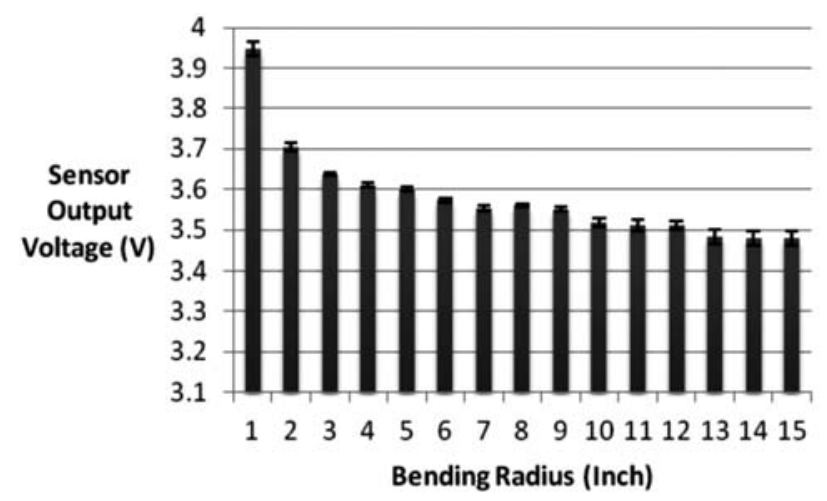

Fig. (4). The calibration of bend sensor: bending radius of curvature versus bend sensor outputs on two-dimensional plane.

There is enough space inside the shaft of the colonoscope for the bend sensor sets with signal wires. When the shaft of the colonoscope bends, it always bends orthogonal to the width of the sensor, due to the composite structure of the sensors and substrate. Thirty channels of signal lines pass through the base of the colonoscope and exit the same side of the biopsy port for connection with the buffer circuits. Each buffer circuit has a variable resistor. The level of the output signal can be individually adjusted for the consistent output of the bending sensor.

In Fig. (3), the thick line depicts the shaft of the colonoscope and variable $r$ and $d$ stand for the bend radius of the loop and three-dimensional loop parameter. When $d$ is closed to zero, the loop is ideally on a two-dimensional plane. Due to the diameter of the shaft $(12 \mathrm{~mm}), d$ cannot be zero but the loop can be treated on the two dimensional plane for the large radius of curvature of the shaft. When the variable $d$ increases, torsion is applied to the bend sensors attached on the substrate and output values are increased.

Fig. (4) shows the calibration results of the single bend sensor without torsion effect $(d=0)$. As bending radius increases, the output voltage is decreasing. The possible minimum bending radius starts from one inch, but it rarely happens under normal configuration of a colon. The maximum bending radius will not exceed ten inches due to the limitation of the shaft length.

Fig. (5) shows the relation of the output voltage between a two and three-dimensional loop. When the variable $d$ in-

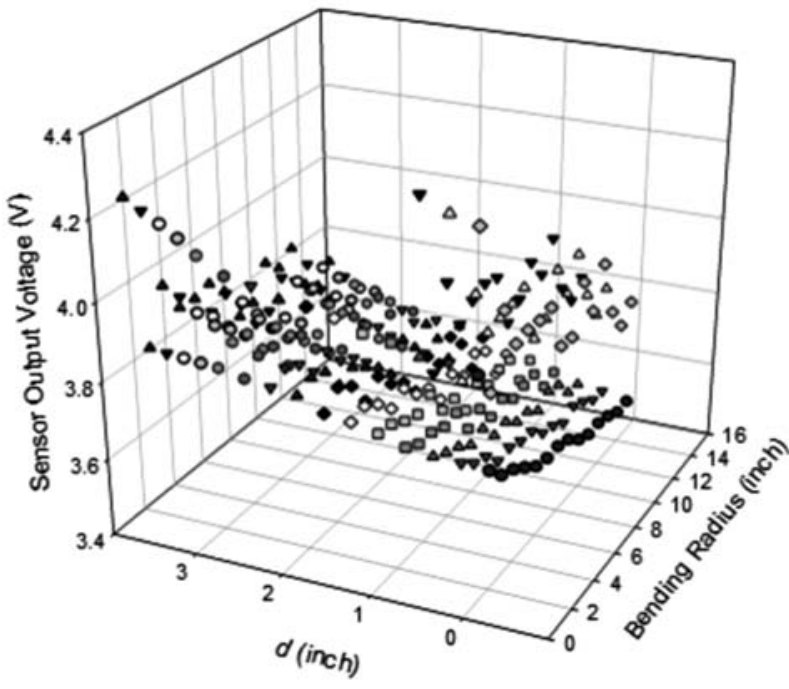

Fig. (5). The transition effect of two to three-dimensional loop.

creases, the loop opens with a small change of bending radius, and the output voltage from the bend sensor is augmented. Since the maximum value of the variable $d$ will not exceed more than four inches, a three-dimensional loop can be projected and assumed to be a simple two-dimensional loop. An N loop can be detected when the shaft of the colonoscope forms two different (or the same) magnitude of hemi-circles. The variable $d$ will be applied to the cases of $\mathrm{N}$ or reversed $\mathrm{N}$ loop, and the detection logic is the same as an alpha or reversed alpha loop.

The system uses the simple loop detection algorithm which projects a three dimensional loop to a two dimensional plane. The thirty channel sensor outputs are connected to the operational amplifiers for impedance matching and signal stability purposes and they are linked to an analog-to-digital converter. LabVIEW is used to collect and analyze all the data for the loop detection. The bending sensors are grouped (three to eight) and the program monitors continuous bending status of each bending sensor. If the summation of the consecutive bend sensor outputs exceeds the designated threshold values, the software detects the loop. If more than one group of sensors is detected, the smaller bending sensor group will be selected over the other group to detect a smaller loop. The Active Colonoscopy Training Model (ACTM) was used to test the bending detection system. The ACTM can measure the forces exerted from the colonoscope to each part of the colon and is capable of localizing the distal tip of the colonoscope. In this experiment, the configuration of the shaft is recorded for validation of the loop detection system.

\section{RESULTS}

Fig. (6) shows the intubation process in the ACTM. The distal tip is advanced in the sigmoid colon in Fig. (6a), ascending colon in Fig. (6b), descending colon in Fig. (6c), and cecum in Fig. (6d). During the intubation process, no significant loop occurred, and the summation of bending sensor groups was under the threshold values. The corresponding values of bending sensors are shown in Fig. (7). No continuous high voltages (around 4 volts) among the consecutive sensor sets were found in the graph, and sparse pick voltages were presented. 


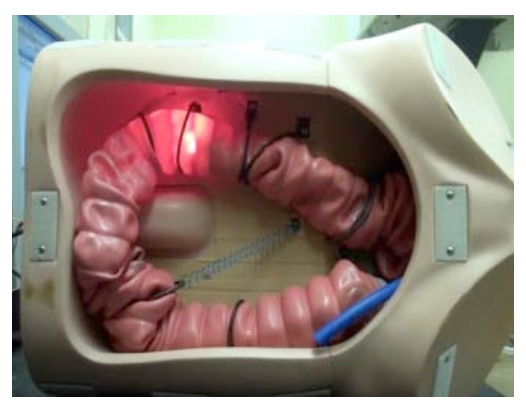

(a)

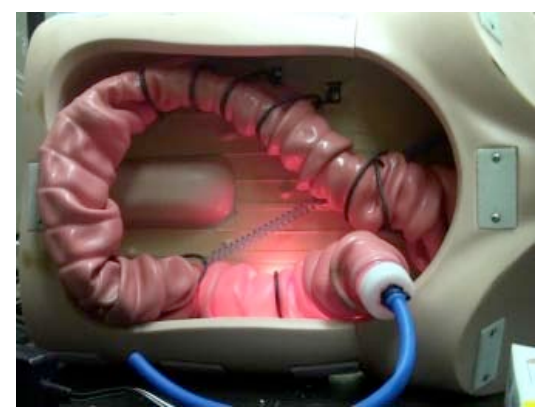

(c)

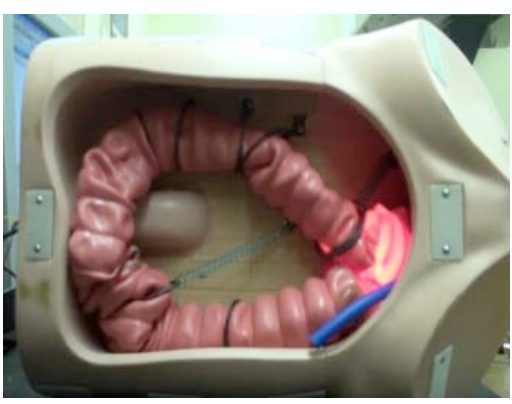

(b)

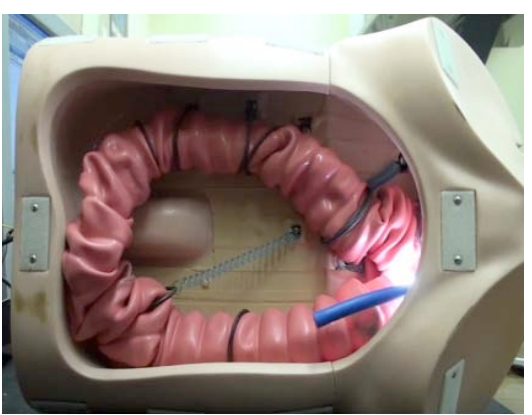

(d)

Fig. (6). Intubation process in the Active Colonoscopy Training Model without significant looping. Anus, descending colon, transverse colon, ascending colon are at nine, twelve, three, and six o'clock position.

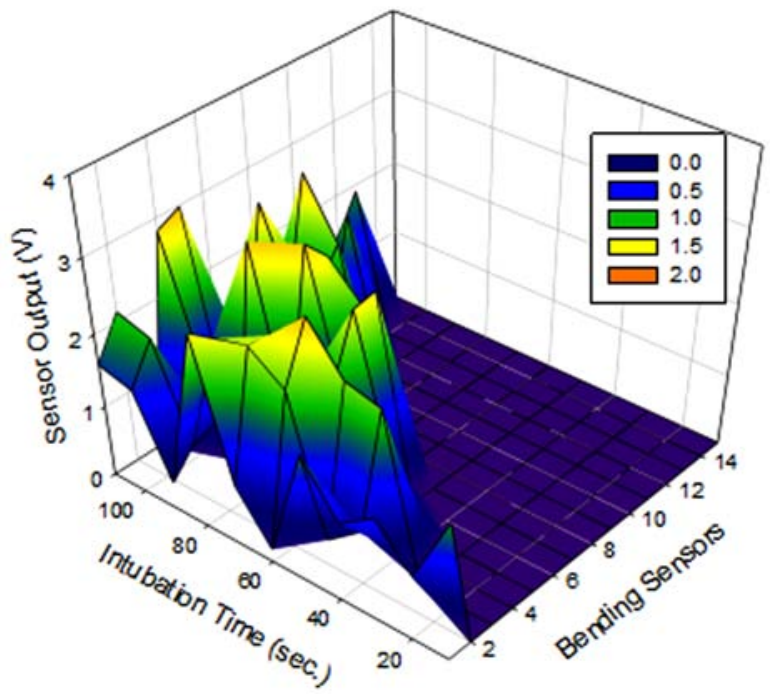

Fig. (7). Bending sensor values during the intubation process without significant looping.

Before the intubation process, the shaft of the colonoscope was originally bent and laid on an auxiliary table or the patient's bed. When the bending sensors inside the shaft of the colonoscope passed through the anus of the ACTM, they were activated and the output signals were processed for loop detection. In Fig. (7), roughly the right side of the $x-y$ plane which presents the bending sensors versus the intubation time remained at zero output voltages, and the fifteenth bending sensor set was activated from the intubation time of 105 seconds.

Fig. (8) shows the intubation process with an N-loop. In Fig. (8a), the distal tip of the colonoscope is located in the descending colon and the N-loop began to form from the anus to sigmoid colon. Because of the N-loop at the sigmoid colon, the advancement of the distal tip was a bit slow and more pressure was exerted on the rectum and sigmoid colon. Fig. (8b) illustrates a continuous N-loop at the sigmoid colon and the distal tip passed Hepatic flexure. At this point, the distal tip cannot be advanced without external help, such as abdomen pressure from an assistant. The advancement trial increased the stress at the rectum and sigmoid colon. Fig. (9) demonstrates the corresponding bending sensor values through the intubation process with the N-loop. The group of consecutive high voltages means the bending of the shaft and two high voltage groups show the $\mathrm{N}$ loop in the Fig. (9). The group of bending sensors were activated and lasted until the shaft of the colonoscope was straightened. Sensor values were not recorded for the retraction process. 


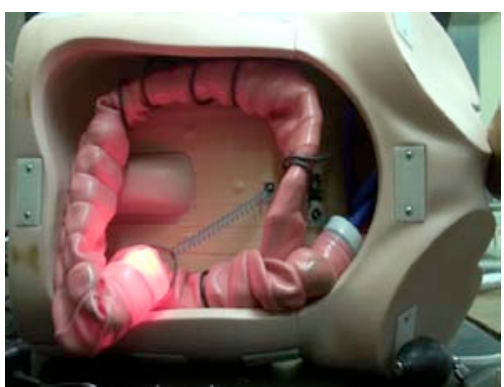

(a)

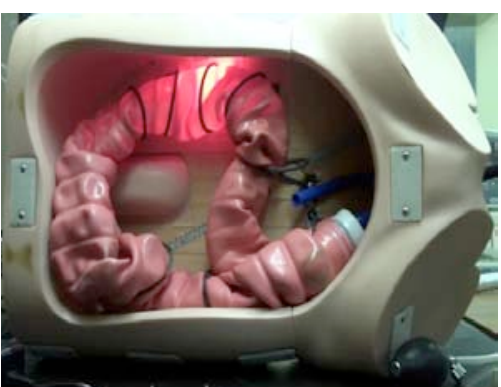

(b)

Fig. (8). Intubation process in the Active Colonoscopy Training Model with looping: (a) N-loop is beginning to occur at the sigmoid colon. The distal tip is located in the descending colon. (b) Due to the N-loop, the distal tip cannot advance in the ascending colon. Abdomen pressure is required for the complete intubation.

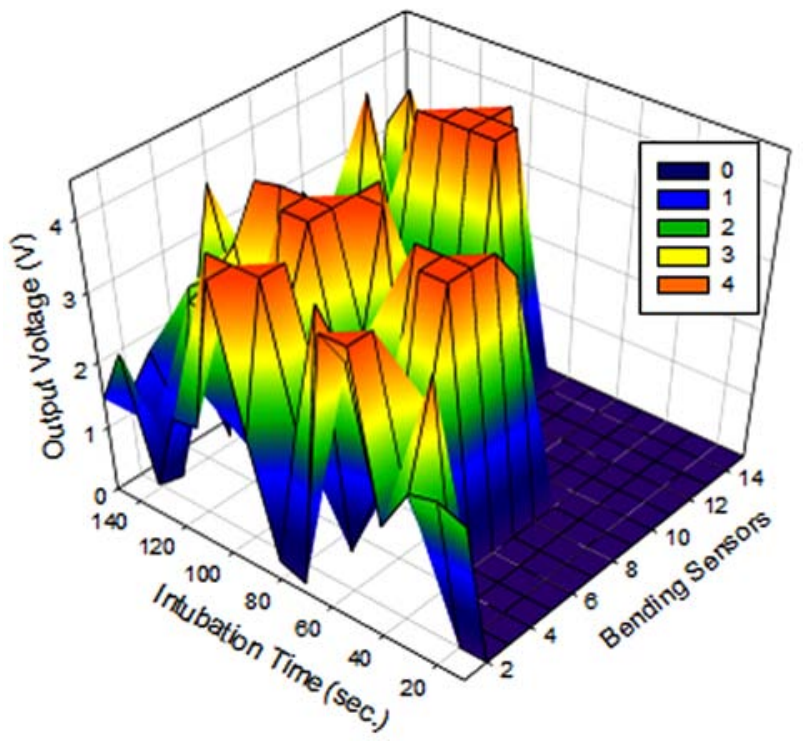

Fig. (9). Bending sensor values during the intubation process with the looping.

\section{DISCUSSION}

The results show that an N-loop is detected during the second intubation process when the distal tip passes the descending colon. No loop was found in the first trial. Generally, the shape of looping depends on the configuration of the colon [39]. The sigmoid colon is the first part to form an alpha or $\mathrm{N}-$ loop. There was no loop for the short length and straight configuration of the sigmoid colon. The intubation time with looping was extended because the colonoscopist consumed time frequently checking for the presence of looping.

For the relatively large bending radius (above 2 inches), the projection of the three-dimensional spiral bending curve reflects well for a two dimensional bending curve. The Nloop was detected throughout the intubation process compared to the small bending radius of alpha loop. If the bending radius of the shaft becomes less than one inch and the distance between the beginning and end points of the loop have a large distance, the bending sensors inside the shaft of the colonoscope are exerted to both torsion and the bending moment. In spite of the pre-calibration of the bending sensors, excessive torsion will distort the output values of the bending sensors, and the loop detection signals will be activated prior, to reaching the designated threshold values.
Sometimes, a significant amount of retraction is necessary to escape looping, especially for the lower part of the colon, regardless of the loop detection [40]. In that case, the colonoscopist needs to maintain the straight configuration of the shaft, especially at the lower colon (rectum and sigmoid colon) to overcome looping at the transverse colon [41].

The display system could show the rough configuration of the shaft on the two-dimensional plane by using the position information. However, too much visual information can distract the colonoscopist causing him to lose focus and potentially miss colorectal disease on the main monitor. Minimal visual and sound warnings will minimize the distraction and maximize the efficiency of the colonoscopy procedure. Colonoscopists with different levels of proficiency before utilizing the system require different levels of experience with the model to work through the learning curve. When evidence of looping is detected, the colonoscopist has to perform various maneuvers, such as retracting, twisting, or advancing the shaft of the colonoscope, to eliminate the looping. A more effective way to shorten the intubation time is to prevent the loop before it happens. In many cases, once the looping forms, it is hard to escape without significant amount of shaft retraction and reinsertion, without guarantee looping will not recur. A key function of the looping detec- 
tion system is to provide early warning of impending loop formation. More case studies with various configurations of the colon are needed to quantify the post looping scenarios for colonoscopists with different levels of expertise.

\section{CONCLUSION}

Looping is one of the major contributors to prolonged intubation during colonoscopy. Various loop configurations were detected by using bending sensors in the shaft of the colonoscope, a data acquisition system and software. By using the bending detection system, the colonoscopist need not know the colonoscope configuration to detect and prevent looping. By understanding the factors that contribute to looping and the pre-warning signs, looping can be avoided or minimized during the colonoscopy. The prototype of this looping detection system showed potentials and limitations. Based on the database of the bending sensors, a more precise pre-looping warning algorithm can be developed, which will be very helpful to minimize looping. The looping detection system showed the coverage limitation of two-dimensional sensors. Maintenance of thirty bending sensors can be an issue due to the durability of the sensors. More parametric interpolation and compensation will be needed to overcome the structural simplification of bending sensors.

\section{CONFLICT OF INTEREST}

The authors confirm that this article content has no conflicts of interest.

\section{ACKNOWLEDGEMENT}

Declared none.

\section{REFERENCE}

[1] Sato K, Fujinuma S, Sakai Y. Original Article Evaluation of the looping formation and pain during insertion into the cecum in colonoscopy. Gastrointest Endosc 2006; 18(3): 181-7.

[2] Mitchell RM, McCallion K, Gardiner KR, Watson RG, Collins JS. Successful colonoscopy; completion rates and reasons for incompletion. Ulster Med J 2002; 71: 34-7.

[3] Shah SG, Saunders BP, Brooker JC, Williams CB. Magnetic imaging of colonoscopy: an audit of looping, accuracy and ancillary maneuvers. Gastrointest Endosc 2000; 52: 1-8.

[4] Eickhoff A, Pickhardt PJ, Hartmann D, Riemann JF. Colon anatomy based on CT colonography and fluoroscopy: impact on looping, straightening and ancillary manoeuvres in colonoscopy. Dig Liver Dis 2010; 42(4): 291-6.

[5] Park DI, Kim HJ, Park JH, et al. Factors affecting abdominal pain during colonoscopy. Eur J Gastroenterol Hepatol 2007; 19(8): 6959.

[6] Kim WH, Cho YJ, Park JY, Min PK, Kang JK, Park IS. Factors affecting insertion time and patient discomfort during colonoscopy. Gastrointest Endosc 2000; 52(5): 600-5.

[7] Elphick DA, Donnelly MT, Smith KS, Riley SA. Factors associated with abdominal discomfort during colonoscopy: a prospective analysis. Eur J Gastroenterol Hepatol 2009; 21(9): 1076-82.

[8] Petersen CR, Adamsen S, Gocht-Jensen P, Arnesen RB, HartHansen O. Splenic injury after colonoscopy. Endoscopy 2008; 40: 76-9.

[9] Sarhan M, Ramcharan A, Ponnapalli S. Splenic injury after elective colonoscopy. JSLS 2009; 13(4): 616-9

[10] Shankar S, Rowe S. Splenic injury after colonoscopy: case report and review of literature. Ochsner J 2011; 11(3): 276-81.

[11] Livstone EM, Kerstein MD. Serosal tears following colonoscopy. Arch Surg 1976; 111(1): 88.

[12] Sjogren RW, Heit HA, Johnson LF, Gremillion DE, Butler ML, Cammerer RC. Serosal laceration: a complication of intra-operative colonoscopy explained by transmural pressure gradients. Gastrointest Endosc 1978; 24(5): 239-42.

[13] Tada M, Kizu M, Akasaka Y. A newly improved sliding tube for colonoscopy for keeping the sigmoid colon in a straight condition. Gastroenterol Jpn 1979; 14(1): 11-3.

[14] Mishkin DS, Schroy PC. Double-baloon endoscopy: extending the arm of the gastroenterologist. Gastroenterology 2006; 131(3): 96971.

[15] Gay G, Delvaux M. Double-balloon colonoscopy after failed conventional colonoscopy: a pilot series with a new instrument. Endoscopy 2007; 39(9): 788-92.

[16] Berger WL, Saeian K. Sigmoid stiffener for decompression tube placement in colonic pseudo-obstruction. Endoscopy 2000; 32(1): 54-7.

[17] Raju GS, Pasricha PJ. ShapeLock: a rapid access port for redeployment of a colonoscope into the proximal colon to facilitate multiple polypectomies in a single session. Gastrointest Endosc 2005; 61(6): 768-70.

[18] Rex DK, Khashab M, Raju GS, Pasricha J, Kozarek R. Insertability and Safety of a Shape-Locking Device for Colonoscopy. Am J Gastroenterol 2005; 100(4): 817-20.

[19] Swain P. The ShapeLock system adapted to intragastric and transgastric surgery. Endoscopy 2007; 39(5): 466-70.

[20] Hawari R, Pasricha PJ. Going for the loop: a unique overtube for the difficult colonoscopy. J Clin Gastroenterol 2007; 41(2): 138-40.

[21] Blachar A, Sosna J. CT colonography (virtual colonoscopy): technique, indications and performance. Digestion 2007; 76(1): 3441.

[22] Dickey W. Does fluoroscopic imaging still have a role in colonoscopy? J Clin Gastroenterol 2004; 38(8): 676-9.

[23] Renault KM, Brander DC, Kristensen EH, Christensen M, Ovesen $\mathrm{H}$. The value of using fluoroscopy during colonoscopy. A prospective randomized study. Ugeskr Laeger 2003; 165(24): 2473-7.

[24] Shah SG, Brooker JC, Thapar C, Williams CB, Saunders BP. Patient pain during colonoscopy: an analysis using real-time magnetic endoscope imaging. Endoscopy 2002; 34(6): 435-40.

[25] Shah SG, Thomas-Gibson S, Lockett M, et al. Effect of real-time magnetic endoscope imaging on the teaching and acquisition of colonoscopy skills: results from a single trainee. Endoscopy 2003; 35(5): 421-5.

[26] Coderre S, Anderson J, Rikers R, Dunckley P, Holbrook K, McLaughlin K. Early use of magnetic endoscopic imaging by novice colonoscopists: improved performance without increase in workload. Can J Gastroenterol 2010; 24(12): 727-32.

[27] Baumann UA. Water intubation of the sigmoid colon: water instillation speeds up left-sided colonoscopy. Endoscopy 1999; 31(4): 314-7.

[28] Hsieh YH, Tseng KC, Chou AL. Patient self-administered abdominal pressure to reduce loop formation during minimally sedated colonoscopy. Dig Dis Sci 2010; 55(5): 1429-33.

[29] Oh SY, Sohn CI, Sung IK, et al. Factors affecting the technical difficulty of colonoscopy. Hepatogastroenterology 2007; 54(77): 1403-6.

[30] De Silva AP, Kumarasena RS, Perera Keragala SD, et al. The prone 12 o'clock position reduces ileal intubation time during colonoscopy compared to the left lateral 6 o'clock (standard) position. BMC Gastroenterol 2011; 11: 89.

[31] Jechart G, Messmann H. Indications and techniques for lower intestinal endoscopy. Best Pract Res Clin Gastroenterol 2008; 22(5): 777-88.

[32] Denis B, Weiss AM, Peter A, Bottlaender J, Chiappa P. Quality assurance and gastrointestinal endoscopy: an audit of 500 colonoscopic procedures. Gastroenterol Clin Biol 2004; 28(12): 1245-55.

[33] Chung JI, Kim N, Um MS, et al. Learning curves for colonoscopy: a prospective evaluation of gastroenterology fellows at a single center. Gut Liver 2010; 4: 31-5.

[34] Williams CB, Saunders BP, Bladen JS. Development of colonoscopy teaching simulation. Endoscopy 2000; 32: 901-905.

[35] Mahmood T, Darzi A. A study to validate the colonoscopy simulator. Surgical Endosc 2003; 17: 1583-9.

[36] Eickhoff A, Jakobs R, Kamal A, Mermash S, Riemann JF, van Dam J. In vitro evaluation of forces exerted by a new computerassisted colonoscope (the NeoGuide Endoscopy System). Endoscopy 2006; 38: 1224-9. 
[37] Haycock AV, Youd P, Bassett P, Saunders BP, Tekkis P, ThomasGS. Simulator training improves practical skills in therapeutic GI endoscopy: results from a randomized, blinded, controlled study. Gastrointest Endosc 2009; 70: 835-45.

[38] Haycock A, Koch AD, Familiari P, et al. Training and transfer of colonoscopy skills: a multinational, randomized, blinded, controlled trial of simulator versus bedside training. Gastrointest Endosc 2010; 71: 298-307.
[39] Renehan AG, Painter JE, Bell GD, Rowland RS, O'Dwyer ST, Shalet SM. Determination of large bowel length and loop complexity in patients with acromegaly undergoing screening colonoscopy. Clin Endocrinol (Oxf) 2005; 62(3): 323-30.

[40] Witte TN, Enns R. The difficult colonoscopy. Can J Gastroenterol 2007; 21(8): 487-90.

[41] Zuber-Jerger I, Endlicher E, Gelbmann CM. Factors affecting cecal and ileal intubation time in colonoscopy. Med Klin (Munich) 2008; 103(7): 477-81.

Received: November 27, 2012

Revised: December 20, 2012

Accepted: December 21, 2012

(C) Choi and Drozek; Licensee Bentham Open.

This is an open access article licensed under the terms of the Creative Commons Attribution Non-Commercial License (http://creativecommons.org/licenses/by-nc/3.0/) which permits unrestricted, non-commercial use, distribution and reproduction in any medium, provided the work is properly cited. 